\title{
EL CAMBIO EN SISTEMAS DE CONTROL DE GESTIÓN: ESTUDIO DE CASO EN UNA PYME INDUSTRIAL
}

Lucio Jesús Uc Heredia

Profesor Investigador

Facultad de Contaduría y Administración

de la Universidad Autónoma de Yucatán

lucio.uc@uady.mx

Salvador Marín Hernández

Profesor Titular de Universidad

Departamento de Economía Financiera y Contabilidad

Universidad de Murcia

Campus Universitario de Espinardo

30100 Murcia

e-mail: salvlau@es 



\section{INTRODUCCIÓN}

La línea de investigación, en el ámbito económico-administrativo, enfocada al cambio en los Sistemas de Control de Gestión (SCG), resulta oportuna ante la imparable transformación en la que actualmente se ven inmersas todas las empresas. Los esfuerzos dedicados a analizar las causas que influyen en el dinamismo y evolución de estos sistemas, constituyen hoy día una creciente línea de investigación ${ }^{1}$ denominada "Cambio en Contabilidad de Gestión".

La pertinencia del estudio del cambio en SCG y su relación con el cambio organizativo y social, ha sido puesta de manifiesto en diversos trabajos (Burns y Scapens, 2000; Kasurinen, 2002; Williams y Seaman, 2002; Araújo, 2003; Escobar y Lobo, 2005; Ford y Greer, 2005; Scapens, 2006; Chenhall y Euske, 2007; Busco, Quattrone y Ricabonni, 2007). Sin embargo, la mayor parte de la literatura existente sobre este fenómeno, lo analiza a la luz de la gran empresa (Laitinen, 2001) y desde la perspectiva de la superioridad teórica de una herramienta sobre otra, siendo escasos los estudios, realizados con una perspectiva organizacional y social en empresas de menor dimensión. Trabajos relativos a la gestión de Pequeñas y Medianas Empresas (PYME) destacan la urgencia de desarrollar herramientas específicas para este tipo de empresas (Fernández, Raja y Whalley, 2006; Gumbus y Lussier, 2006); se argumenta que determinadas herramientas pueden ser eficaces para la gran empresa, pero no serlo para una de menor dimensión (Laitinen, 2001).

El interés de este trabajo es contribuir a la literatura existente sobre el cambio en SCG, desde una perspectiva organizativa y social en las PYME. La pregunta de investigación que guía el estudio es ¿qqué factores están involucrados en el cambio en SCG en las PYME industriales manufactureras? Para responder a esta cuestión, se ha establecido como objetivo conocer los factores involucrados en este fenómeno siguiendo el modelo de Kasurinen (2002).

Para lograr el objetivo planteado, se realizó un estudio de caso en una PYME industrial manufacturera del subsector alimentos y bebidas de Yucatán, México. Se consideró esta metodología, porque permite estudiar a profundidad las variables involucradas y, por consiguiente, permite una mejor aproximación hacia la comprensión del cambio y de sus procesos (Araújo, 2003; Azofra, Pietro y Santidrián, 2004; Scapens, 2006; Blanco, 2007).

\footnotetext{
${ }^{1}$ La importancia y actualidad del tema puede corroborarse por la existencia de la Red Europea para la Investigación en el Cambio Organizacional y Contable (ENROAC, por sus siglas en inglés), constituida en el año 1999. A la fecha, la ENROAC ha organizado seis congresos con carácter internacional. En 2007, la revista Management Accountig Research dedicó un número especial al "Cambio en Contabilidad de Gestión" (número 2, volumen 18).
} 
Después de esta introducción, se presenta la segunda sección que contiene la revisión de estudios relativos al cambio en SCG, que constituye el marco de referencia para el desarrollo y discusión del caso; la sección posterior describe la metodología utilizada; seguidamente se presenta la cuarta sección donde se desarrolla el caso estudiado; y finalmente, la quinta sección ofrece una discusión de los resultados del estudio.

\section{EL CAMBIO EN LOS SISTEMAS DE CONTROL DE GESTIÓN}

En un sentido amplio, Simons (1990, p.128) define los SCG como "las prácticas y procedimientos formales basados en información, que los administradores utilizan para mantener o modificar conductas en las actividades de una organización". Desde una perspectiva pragmática, los SCG representan una composición multidimensional de los subsistemas de planeación y control, a través de los cuales se obtiene información para la toma de decisiones administrativas y para la evaluación, y cuyo fin es la mejora del desempeño de una organización (Williams y Seaman, 2002). No obstante, su alcance es mayor ya que ayudan al administrador a coordinar a los integrantes de la organización, a asignar recursos, y en la dirección lo ayudan al logro de la misión, visión, objetivos y metas de la empresa (Ghosh, 2005). De este modo, el sistema se compone de varios subsistemas interrelacionados, pero que en la realidad de las organizaciones pueden implementarse y funcionar de manera independiente, aunque con el menoscabo de los beneficios que se lograrían con su implementación integral (Rotch, 1993; Kanthi, 2007). De acuerdo con lo mencionado anteriormente, herramientas como la planificación formal, la presupuestación, la revisión del ambiente exterior, el análisis de los competidores, los reportes de desempeño y evaluación, los procesos de asignación de recursos, los sistemas de incentivos y recompensas a empleados, la contabilidad financiera, entre otras, funcionando de manera independiente $o$ integral constituyen los SCG de una organización (Simons, 1990; Rotch, 1993; Kanthi, 2007).

En el siglo pasado surgieron diversas innovaciones en los subsistemas que conforman los SCG. Por ejemplo, en la década de los cincuenta surgió la Administración por Objetivos de Drucker, en los ochenta surgió el Costeo Basado en Actividades (ABC, por sus siglas en inglés) de Cooper y Kaplan; en los noventa el Cuadro de Mando Integral (BSC, por sus siglas en inglés) de Kaplan y Norton; y en las últimas décadas, el imparable desarrollo informático hizo posible la creación de poderosas herramientas al integrar el funcionamiento de varios subsistemas de gestión (por ejemplo el Manufacturing Resourcing Planning "MRP" o el Enterprise Resource Planning "ERP”). Blanco (2007) presenta la evolución de los SCG por etapas donde la primera y segunda etapas (1950-1985) consistieron en la reformulación de técnicas ya existentes, la tercera etapa y cuarta etapas 
(1985-1995) fueron adiciones de nuevos instrumentos y la etapa actual (a partir de 1995) ha consistido en la actualización de técnicas, instrumentos y la introducción de valores en esta área.

La evolución de los SCG, ha abierto nuevas interrogantes acerca del papel que juegan en el cambio organizacional, siendo evidente, por sus alcances, que dicha relación existe (Araújo, 2003; Sulaiman y Mitchell, 2005; Blanco, 2007). Sin embargo, el uso por parte de las empresas de las novedosas técnicas, desarrolladas el siglo pasado, es bajo (Garengo, Biazzo y Biticci, 2005; Scapens, 2006). Según algunos investigadores, el carácter conservador de la contabilidad ocasiona que los cambios sean lentos y moderados, en contraposición de frecuentes y radicales (Busco, Riccaboni y Scapens, 2000; Sulaiman y Mitchell, 2005). Incluso, Martínez (2004) afirma que la información contable ayuda o, en su defecto, retrasa a las organizaciones en la detección de la necesidad del cambio y en el establecimiento del modo de acometerlo. Sobre este aspecto, Scapens y Roberts (1993) indican que la resistencia a cambiar se debe a las fallas en el aseguramiento de la legitimidad de los nuevos sistemas y a que no se converge en un lenguaje común entre las áreas de producción y contabilidad.

Adicional al conservadurismo señalado en el párrafo anterior, el cambio en SCG no se presenta como un fenómeno homogéneo en las empresas. Por ejemplo, Simons (1990) menciona que existen diferencias entre los SCG de las empresas que compiten, de acuerdo a la estrategia que siguen; Martínez (2004) indica que las variaciones en el entorno y en las estructuras organizacionales implican cambios en las necesidades de información, viéndose alteradas la cantidad y la naturaleza de la información, así como su utilización en la toma de decisiones y el control. Esta heterogeneidad del cambio en SCG, dificulta su análisis e interpretación.

Desde el punto de vista de la Teoría de la Contingencia, el cambio en SCG se explica como consecuencia de la relación entre elementos del contexto interno y externo de la empresa (Otley, 1980). La Teoría Institucional asume que los SCG se definen de acuerdo a complejas relaciones que ocurren entre las instituciones, y que se traducen en acciones a través de las reglas, rutinas y creencias (Burns y Scapens, 2000). De esta teoría se derivan el Nuevo Institucionalismo Económico y el Nuevo Institucionalismo Sociológico (Scapens, 2006). La Teoría de la Estructuración de Giddens combina factores contingentes e institucionales para explicar la multiplicidad de actores y situaciones que convergen en el cambio en un espacio-tiempo (Araújo, 2003).

En la literatura existente sobre el cambio en SCG se identifican varios factores contingentes relacionados con este fenómeno. Otley (1980) señala que aspectos particulares de un SCG dependerán de las circunstancias específicas de la organización, 
tales como el entorno de la empresa, su tecnología y su estructura organizacional. En el mismo sentido, Burns y Vaivio (2001) atribuyen el cambio a fenómenos como la globalización de los mercados, el incremento de la competencia, los avances tecnológicos y los cambios en las formas de producción. Según estos autores, estos fenómenos han causado que los procesos para la toma de decisiones requieran de múltiple información, cada vez en mayor cantidad y en tiempo real. Dada la importancia de identificar la influencia de estos factores, Haldma y Lääts (2002) clasifican las contingencias que aceleraban o disminuían los cambios de sistemas contables en aspectos ambientales, organizacionales y tecnológicos. Sin embargo, también existen estudios que afirman que el ambiente externo no influye en el cambio de SCG. Baines y Langfield-Smith (2003) examinaron los cambios ocurridos en los SCG como consecuencia de los cambios en el ambiente externo y encontraron que el ambiente cambiante no tiene un impacto directo sobre los SCG.

Otros estudios relacionan el cambio en SCG, específicamente con las modificaciones de las prácticas de producción. Sriram (1995) encontró que las empresas manufactureras para encarar el actual ambiente competitivo están implementando sistemas de producción flexibles. Estos sistemas les proveen de varias ventajas estratégicas; sin embargo, las ventajas estratégicas funcionan solamente si la organización puede mejorar su sistema de información, particularmente el contable y financiero.

Como se ha visto, varios trabajos destacan la importancia de analizar la influencia de los factores y barreras del cambio con la finalidad de controlar sus efectos al realizar el cambio. Para ello, algunos trabajos se han enfocado al desarrollo de modelos que permitan un análisis sistemático de estos factores. Innes y Mitchell (1990) dividen los factores asociados al cambio de SCG en tres categorías: motivadores, catalizadores y facilitadores. Denominaron "motivadores" a los factores que impulsan de manera general el cambio, "catalizadores" a las necesidades más especificas asociadas con el cambio y "facilitadores" a los factores que apoyan el cambio pero que no tienen la suficiente fuerza para provocarlo, sin embargo son necesarios para que ocurra. Cobb, Helliar e Innes (1995) añadieron a este modelo la influencia del líder (reconociendo el papel de los individuos en el cambio), el momentum y las barreras del cambio (factores que obstaculiza, atrasan e incluso evitan el cambio).

Siguiendo los modelos de Cobb et al. (1995), Kasurinen (2002) subdividió las barreras del cambio en tres subcategorías a las que llama confusores, frustadores y retrasadores. Este autor consideró que al detallar y clasificar las barreras del cambio, era posible aumentar su comprensión y, por tanto, se facilitaba la búsqueda de soluciones para superarlas. El modelo integrado propuesto por Karusinen (2002) se muestra en la figura 1. 
Figura 1. Modelo integrado del cambio en SCG

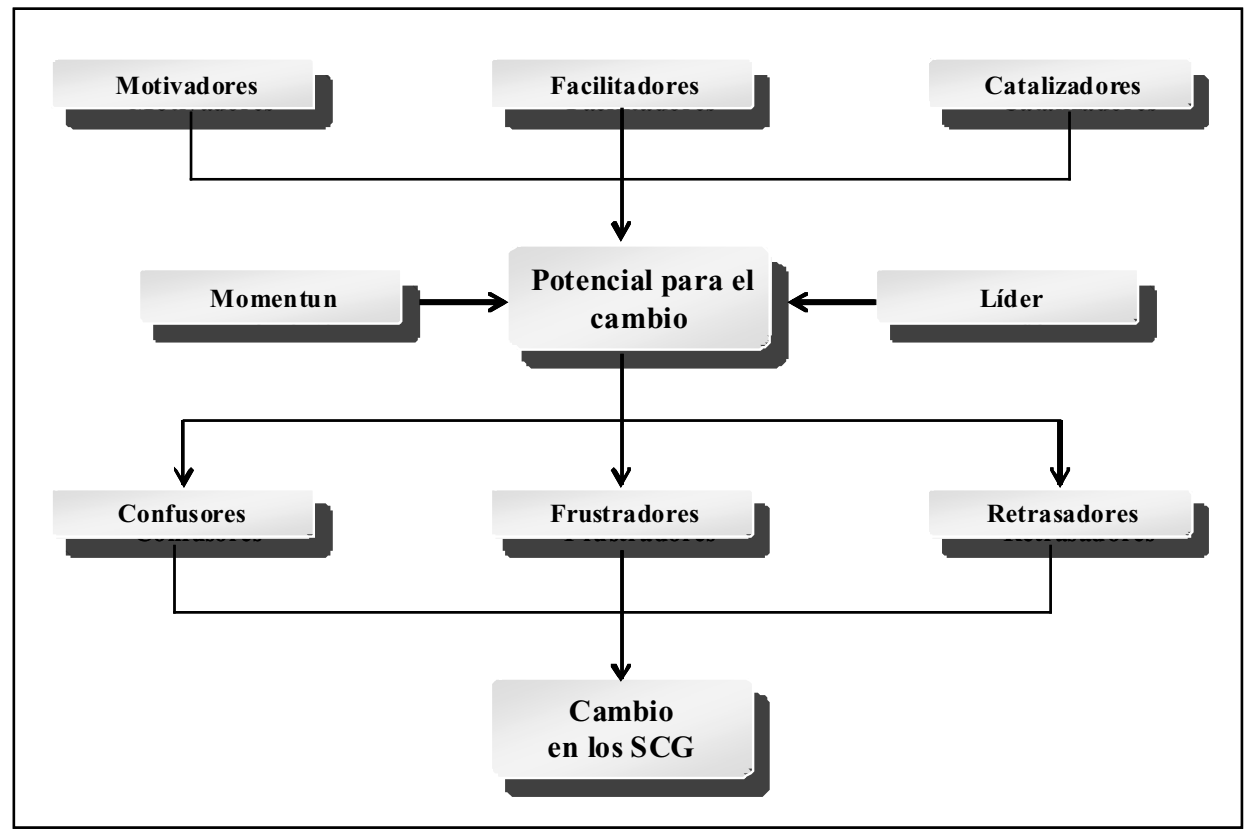

Fuente: Karusinen (2002).

El estudio del cambio en SCG, se ha desarrollado principalmente en la gran empresa. Para empezar a construir la teoría para la PYME, es necesario ir abordando los casos de éxito y fracaso en la adopción de nuevas herramientas o en la modificación de las existentes en este tipo de empresas (Gumbus y Lussier, 2006). En cualquier organización, las decisiones deben sustentarse con información. Por tanto, para el control de la gestión se hace necesario un sistema formal que recoja, elabore y comunique información (Somohano y Martínez, 2003).

Las PYME no deben resignarse a la perdida de relevancia en el contexto empresarial y permanecer estáticas ante estos acontecimientos. El actual escenario mundial les exige nuevas formas de obtener y procesar la información adecuada para alcanzar una gestión efectiva. Sin embargo, el desarrollo de herramientas de gestión ocurre en países económicamente desarrollados, mientras que los demás países adoptan o adaptan estas técnicas dependiendo del grado de difusión que dichas técnicas puedan lograr en sus comunidades contables. Un fenómeno que llama la atención es que algunas organizaciones permanezcan estables respecto a estos temas, a pesar de los embates generados por los cambios en el ambiente. En el caso de Latinoamérica no existe suficiente evidencia empírica que permita 
comprender el fenómeno del cambio en SCG en el contexto de la PYME industrial manufacturera. Por ello el presente estudio pretende analizar este fenómeno de manera sistemática basándose en los referentes teóricos analizados en esta sección.

\section{DISEÑO DEL ESTUDIO DE CASO}

Para alcanzar el objetivo de conocer los factores involucrados en el cambio en SCG en una PYME industrial manufacturera, de acuerdo al modelo de Kasurinen (2002), se diseñó el estudio empírico con un enfoque cualitativo. Este enfoque es recomendado por autores como Araújo (2003), Ayuso y Ripoll (2005), Scapens (2006) y Blanco (2007) cuando se tiene como objetivo la comprensión de un fenómeno en organizaciones específicas. Es útil porque permite el análisis detallado de los casos de éxito o fracaso en la creación, adopción o adaptación de las herramientas que conforman los SCG en las PYME y con ello se logra el avance en la comprensión de este fenómeno para este tipo de empresas (Gumbus y Lussier, 2006).

Para la realización de este estudio se seleccionó como población objetivo a las PYME industriales manufactureras del sub-sector de alimentos y bebidas de Yucatán, México. La importancia de seleccionar a las PYME de Yucatán, radica en que este estado se encuentra entre las 10 regiones mexicanas de crecimiento más rápido y que a pesar del rezago que presenta en comparación con otros estados, también se ubica como una de las regiones más dinámicas (OCDE, 2008). La información de los Censos Económicos 2004 (INEGI, 2006) indica que el sector manufacturero en México es el más importante en la generación de producción bruta total, $43.3 \%$, concentrando $10.9 \%$ de las unidades económicas y una de cada cuatro personas ocupadas. Desde 1995, la participación de la industria de alimentos y bebidas en Yucatán ha superado el 50.0 \% del total del valor aportado por el sector manufacturero de la entidad y posee el $25 \%$ de la inversión total en manufactura del estado, lo que pone de manifiesto la importancia de la industria a nivel local (INEGI, 2006). Por ello, el sub-sector alimentos y bebidas es idóneo para examinar cómo han cambiado sus prácticas de gestión, siendo el interés principal enfocarse a las de menor tamaño por el peso que tienen en el tejido empresarial ${ }^{2}$. La obtención de información se realizó durante el periodo de noviembre 2008 a febrero de 2009.

Dado que el cambio en SCG no es un fenómeno común y no existen estadísticas sobre qué empresas han cambiado sus sistemas en la región, la selección del caso fue teórica, a partir de las recomendaciones de profesionales de gestión externos (consultores, auditores y contadores).

\footnotetext{
${ }^{2}$ Las empresas grandes representan sólo $0.9 \%$ del total de unidades económicas de este sector en el país (INEGI, 2006)
} 


\section{DESARROLLO DEL CASO}

El estudio empírico se desarrolló en una PYME industrial dedicada a la elaboración y comercialización semi-industrial de panes y pasteles para el mercado local, y que en adelante se le identificará como La Empresa ${ }^{3}$. Esta empresa, de carácter familiar y capital 100\% local, es dirigida por el propietario fundador, que a su vez es accionista mayoritario. Su forma de producción puede clasificarse como industrializada pero con mano de obra intensiva, porque ninguno de sus procesos están automatizados. El personal ocupado por esta empresa, es de aproximadamente 80 trabajadores repartidos en tres grandes áreas: producción, ventas y administración. A lo largo de más de dos décadas de funcionamiento La Empresa ha permanecido en el competitivo mercado local, enfrentando una competencia caracterizada por la producción automatizada y con medios de distribución del producto cada vez más efectivos; para enfrentar esta competencia, el director de esta PYME ha enfocado su estrategia hacia la innovación en productos y ha realizado importantes esfuerzos en mercadotecnia para posicionar su imagen en el mercado. Ante la imposibilidad de distribuir sus productos a través de intermediarios, han abierto varios puntos de ventas con la intención de incrementar el desplazamiento de sus productos, lo que organizativamente ha significado un crecimiento del negocio y ha ocasionado la necesidad de un control efectivo de dichos puntos de ventas.

El cambio en los SCG de La Empresa ha sido impulsado y liderado por el fundador/director de la empresa. Su visión empresarial y la comprensión del negocio, lo condujo a valorar la importancia de contar con sistemas de información formales para conducir sus estrategias. Por ello, asesorado por consultores externos, elaboró un plan estratégico para identificar sus principales problemáticas. Estas problemáticas se jerarquizaron y se destacó la necesidad de contar con información en tiempo real de sus puntos de ventas.

El cambio del SCG se relaciona con la apertura de puntos de ventas (necesidad de un mejor control del crecimiento de la organización), la incursión de nuevos productos (para hacer frente a la fuerte competencia del sector) y por la profesionalización de su administración, que también incluye a familiares. Este cambio, afectó la estructura organizacional de La Empresa; la transformación inició concretamente en los puntos de venta (precisamente en donde el manejo de personal es clave) y poco a poco, ha abarcado otras áreas. El cambio se materializó con la implantación de un sistema de información que desde los puntos de

${ }^{3}$ Por motivos de confidencialidad no se revela el nombre de la empresa, ni se detallan aspectos técnicos de las innovaciones en SCG realizadas. 
ventas provee información en tiempo real a los encargados de la planificación de la producción diaria y de la determinación de costos de producción. También se implantó un sistema semi-integrado de gestión que con ciertas limitaciones proporciona información económico-financiera con diferentes niveles de agregación. Ambos sistemas son desarrollos propios de la empresa y significan un cambio de importancia moderada, aunque todavía trabajan en su mejora. A la fecha de este estudio, la contabilidad financiera y el sistema de costos coexisten sin cambios con los nuevos sistemas de información.

La observación directa permitió determinar que el directivo está más centrado en su papel de dirigir y se enfoca menos a las tareas, además reconoce las limitaciones de la información con la que cuenta, se relaciona con asesores profesionales, con otros empresarios y está predispuesto a aprender. El establecimiento de estrategias formales y la visión más amplia del negocio, así como la existencia de planificación formal, conducen a la necesidad de cambiar los SCG existentes por sistemas más adecuados para la organización. Ante la inexistencia de un SCG específico para sus necesidades (se realizaron intentos infructuosos con sistemas prediseñados con importantes pérdidas económicas), se trazó el objetivo de mejorar los existentes (en principio no han pretendido un cambio radical en los SCG), a través del desarrollo informático de sus sistemas manuales. La información que se considera prioritaria se genera en los niveles más bajos de la jerarquía, lo que ha obligado al director a buscar la cooperación de estos niveles para lograr el buen funcionamiento del sistema. Mediante diversos cursos de formación permanente, se ha logrado que los empleados entiendan y valoren el uso del sistema, incentivados por las recompensas que obtienen al ser evaluados.

El proceso no ha sido tarea fácil. El director señala la dificultad para encontrar consultores expertos. Adicionalmente, los profesionales egresados de las principales carreras empresariales adolecen de la falta de preparación y experiencia en estos temas. Por otro lado, la inversión en la formación del personal ha supuesto una inversión económica importante. A través de la inversión en capacitación y entrenamiento de personal, y mediante pruebas de ensayo y error, han logrado seleccionar aquellas herramientas de control de gestión que le son más adecuadas. Los factores y barreras relacionados con estos cambios se presentan en la figura 2, siguiendo la clasificación de Karusinen (2002). 
Figura 2. Modelo del cambio en SCG para La Empresa

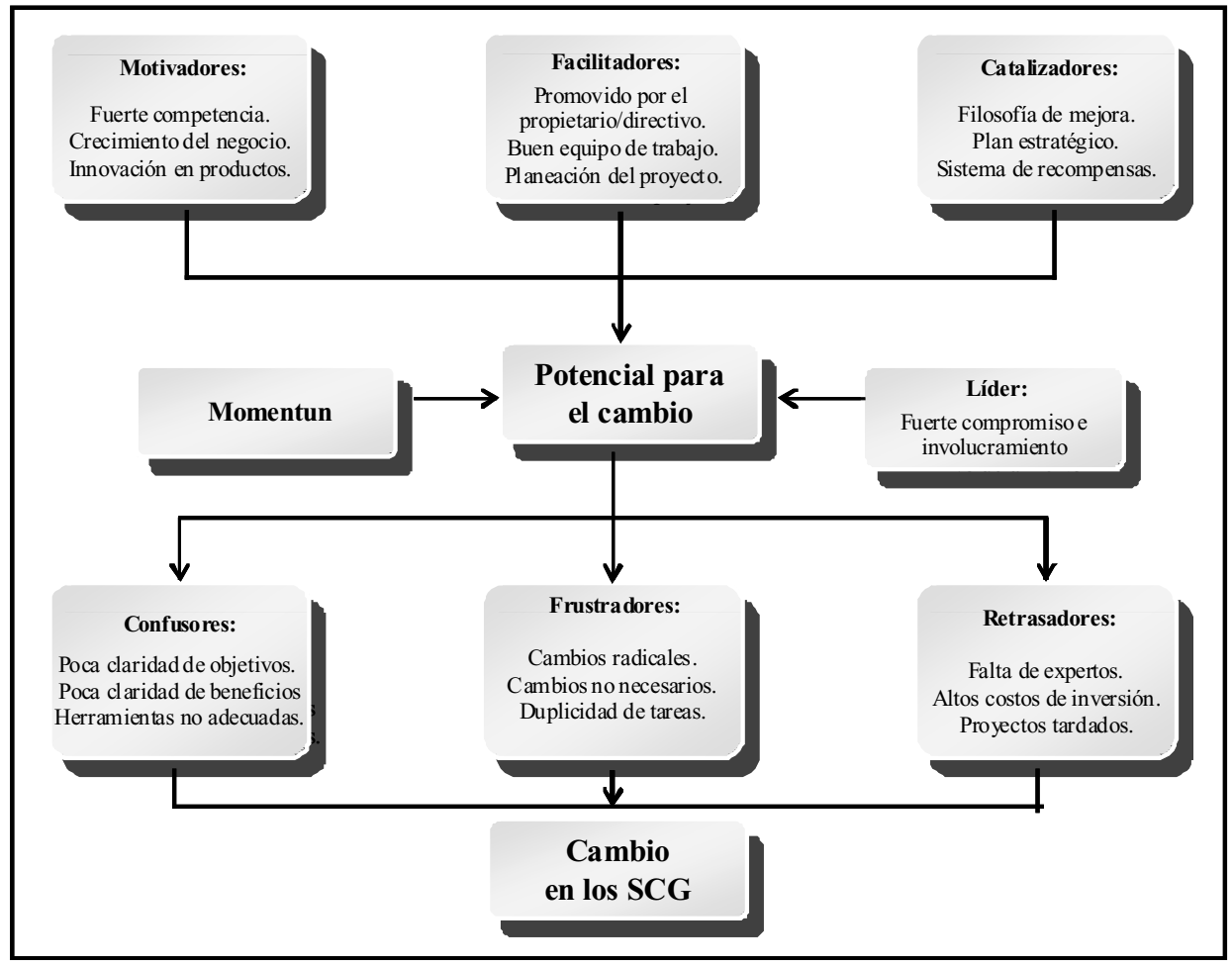

Fuente: elaboración propia con base en el modelo de Karusinen (2002).

\section{DISCUSIÓN}

La industria de alimentos y bebidas de Yucatán, México, es actualmente un sector de rápido crecimiento que concentra una parte importante de la inversión en manufactura de la región. Esto lo ubica como un sector de importancia estratégica para el Estado. No obstante, las empresas locales del sector presentan un gran rezago y se enfrentan a grandes desafíos. El estudio presentado tuvo como objetivo responder la cuestión de ¿qué factores están involucrados en el cambio en SCG en las PYME industriales manufactureras? Para ello, se utilizó el modelo integrado de cambio en SCG de Karusinen (2002) en una PYME industrial manufacturera del sub-sector alimentos y bebidas.

En principio, el estudio permite observar que el cambio en SCG está íntimamente ligado a la visión y deseos del director o administrador. El papel del directivo 
como responsable de la adopción de SCG en las PYME, es un tema estudiado por autores como Maes et al. (2005) y Garengo et al. (2005). Estos autores, coincidentemente con los resultados obtenidos en este trabajo, señalan que los directores tienen un papel importante en la selección de los SCG pero únicamente cuando están plenamente convencidos de sus beneficios. Adicionalmente, el directivo juega el papel de líder del proyecto y se pudo corroborar en este estudio, que su compromiso como responsable del cambio es fundamental para realizarlo con éxito, en el mismo sentido han concluido Argyris y Kaplan (1994), Cobb et al. (1995), Martínez y Heredia (2003) y Vélez, Sánchez y Araújo (2005).

Los argumentos señalados como motivadores del cambio en SCG se refieren a aspectos contingentes (Otley, 1980) e institucionales (Burns y Scapens, 2000). Entre los aspectos ambientales que motivaron el cambio en los SCG, destacan el establecimiento de estrategias para contrarrestar la fuerte competencia y el crecimiento que causa la complejidad del negocio, similares resultados han reportado Haldma y Lääts (2002), Street y Meister (2004) y Martínez (2004). Los aspectos organizacionales más influyentes en la innovación de los SCG encontrados, son la necesidad de un lenguaje común para el establecimiento de objetivos, metas, incentivos y recompensas (Haldma y Lääts, 2002; Martínez 2004) y el cambio en la estructura de la organización (Haldma y Lääts, 2002).

Estos aspectos ejercen una fuerte presión para realizar el cambio en SCG, pero por la lentitud con el que el cambio sucede tampoco pueden relacionarse con el momento de tomar la decisión de realizar el cambio. Aún cuando la planeación estratégica coloca el cambio en SCG como un objetivo urgente, parece ser que el convencimiento progresivo del directivo juega un papel crucial para apurar la innovación del SCG. Así, la falta de convencimiento del directivo y los recursos limitados, en lo que se refiere al personal y financiero, constituyen las principales barreras del cambio. En sentido contrario, se puede relacionar la capacidad de aprendizaje del directivo con la innovación en herramientas para gestionar su empresa. Una vez superadas y controladas las barreras del cambio, los SCG siguen una configuración basada en las dimensiones del estructuralismo contingente como las señala Araújo (2003), es decir, adquieren significación, dominación y legitimización. La significación es la dimensión cognoscitiva mediante la cual los agentes se comunican y se entienden entre sí; la dominación se refiere a la capacidad de un sistema social de alcanzar resultados, es decir, de producir poder; y la legitimización es cuando se constituyen como medios a través de los cuales se legitiman las acciones de los miembros de la organización, señalando o estableciendo ideas sobre lo que es y no es importante, y sobre lo que debe hacerse o justificarse, y controlándolo, institucionalizando así las actuaciones de los actores. 
Este trabajo concluye haciendo el señalamiento de la imperante necesidad de construir un modelo de gestión específico para la PYME, sobre todo en el actual contexto empresarial, altamente competitivo y cambiante. Dicho modelo debe ser diseñado para adaptarse a la diversidad empresarial que caracteriza a la PYME, pero a la vez debe ser lo suficientemente homogéneo para facilitar su adopción. Es decir, una herramienta de gestión que en naturaleza sea heterogénea, pero que en su difusión y práctica sea homogénea. Así, se tendría una herramienta común que permita a los propietarios/directores de la PYME, experimentar su uso $\mathrm{y}$ adaptarla a sus necesidades. Este trabajo ha analizado a una PYME que ha realizado un cambio considerado exitoso, pero aún falta analizar a las que permanecen sin cambios. Aspectos como el papel de los asesores externos (independientes o agencias gubernamentales), la pasividad de las asociaciones empresariales y de los contadores (incluso sus asociaciones) o la escasa vinculación empresa-universidad, abren nuevas posibilidades de investigación. Específicamente las asociaciones empresariales y las agencias de gobierno deben animar a la PYME para innovar en sus SCG. La universidad debe modificar sus planes y programas para egresar profesionales con un fuerte conocimiento aplicado de estos sistemas y los colegios de profesionales deben insistir en la difusión y discusión de sistemas avanzados de gestión entre los contadores agremiados.

\section{REFERENCIAS}

Araújo, P. (2003): “Nuevo institucionalismo, teoría de la estructuración y cambio en los sistemas y prácticas de contabilidad de gestión: Teorías y métodos de investigación", Revista Española de Financiación y Contabilidad, Vol. XXXII, No. 118, julio-septiembre 2003, pp. 693-724.

Argyris, C. y Kaplan, R. (1994): "Implementing new knowledge: the case of activity-based costing, Accounting Horizons, Vol. 8, pp. 83-105.

Ayuso, A., y Ripoll, V. (2005): "El estudio de casos como prototipo de la investigación en contabilidad de gestión desde una perspectiva cualitativa", Revista Iberoamericana de Contabilidad de Gestión, Vol. 3, No. 5, pp.131-168.

Azofra, V.; Prieto, B. y Santidrián, A. (2004): "Verificación empírica y método del caso: revisión de algunas experiencias en contabilidad de gestión a la luz de su metodología”, Revista Española de Financiación y Contabilidad, Vol. XXXIII, No. 121, abril-junio 2004, pp. 349-377.

Baines, A. y Langfield-Smith, K. (2003): "Antecedents to management accounting change: a structural equation approach", Accounting, Organizations and Society, Vol. 28, pp. 675-698. 
Blanco, I. (2007): “Contabilidad de gestión. Tópicos y agenda de investigación”, Polytechnical Studies Review, Vol. IV, No. 7, pp. 41-88.

Burns, J. y Scapens, R. (2000): “Conceptualizing management accounting change: an institutional framework", Management Accounting Research, Vol. 11 , pp. 3-25.

Burns, J. y Vaivio, J. (2001): "Management accounting change", Management Accounting Research, Vol. 12, pp. 389-402.

Busco, C.; Quattrone, P. y Riccaboni, A. (2007): "Management Accounting Issues in interpreting its nature and change", Management Accounting Research, Vol. 18, pp. 125-149.

Busco, C.; Riccaboni, A. y Scapens, R. (2000): "Management Accounting Systems and Organisational Culture: an Institutional Framework for Interpreting their linkages and Process of Change", Paper prepared for the 2nd Workshops on Management Accounting Change, Oslo, Norway, 11-14 May 2000.

Chenhall, R. y Euske, K. (2007): "The role of management control systems in planned organizational change: An analysis of two organizations", Accounting, Organizations and Society, Vol. 32, pp. 601-637.

Cobb, I.; Helliar, C. e Innes, J. (1995): "Management accounting change in a bank", Management Accounting Research, Vol. 6, pp. 155-175.

Escobar, B. y Lobo, A. (2005): "Una revisión sobre cambio organizativo: Implicaciones para la contabilidad de gestión”, Universidad Eafit, Vol. 41, No. 137, enero-marzo 2005, pp. 96-122.

Fernandes, K.; Raja, V. y Whalley, A. (2006): "Lessons from implementing the balanced scorecard in a small and medium size manufacturing organization”, Technovation, Vol. 26, pp. 623-634.

Ford, M. y Greer, B. (2005): "The Relationship between Management Control System Usage and Planned Change Achievement: An Exploratory Study", Journal of Change Management, Vol. 5, No. 1, 29-46.

Garengo, P.; Biazzo, S. y Biticci, U. (2005): "Performance measurement systems in SMEs: A review for a research agenda", International Journal of Management Reviews, Vol. 7, No. 1, pp. 25-47. 
Ghosh, N. (2005): Management Control Systems, Prentice Hall of India, India.

Gumbus, A. y Lussier, R. (2006): "Entrepreneurs Use a Balanced Scorecard to Translate Strategy into Performance Measures", Journal of Small Business Management, Vol. 44, Núm. 3, pp. 407-425.

Haldma, T. y Lääts, K. (2002): "Influencing contingencies on management accounting practices in Estonian manufacturing companies", University of Tartu, Faculty of Economics and Business Administration, No. 13.

INEGI (2006): Micro, Pequeña, Mediana y Gran Empresa. Estratificación de los Establecimientos. Censos Económicos 2004, Instituto Nacional de Estadística, Geografía e Informática, 2006, México, DF.

Innes, J. y Mitchell, F. (1990): "The process of change in management accounting: some field study evidence", Management Accounting Research, Vol. 1, pp. 3-19.

Kanthi, S. (2007): “A framework for management control research", Journal of Management Development, Vol. 26, No. 9, pp. 895-915.

Kasurinen, T. (2002): "Exploring management accounting change: the case of balanced scorecard implementation”, Management Accounting Research, Vol. 13, pp. 323-343.

Laitinen, E. (2001): "Management accounting change in small technology companies: towards a mathematical model of the technology firm", Management Accounting Research, Vol. 12, pp. 507-541.

Martínez, M. (2004): "Proceso, antecedentes y consecuencias de la adopción de innovaciones contables: contrastación de un modelo integrador", Cuadernos de Economía y Dirección de la Empresa, Núm. 21, pp. 5-26.

Martinez, M. y Heredia, J. (2003): "El balanced scorecard. Estudio del proceso de implantación en una mediana empresa", Revista Iberoamericana de Contabilidad de Gestión, Vol. 1, No. 2, pp. 1-14.

OCDE (2008): Estudios Territoriales de la OCDE Yucatán, México, Fundación Plan Estratégico de Mérida- Organisation for Economic Co-operation and Development, para la edición en español, México, 2008. 
Otley, D. (1980): "The contingency theory of management accounting: Achievement and prognosis", Accounting, Organizations and Society, Vol. 5, pp. $413-428$

Rotch, W. (1993): "Management Control Systems: One View of Components and Their Interdependence", British Journal of Management, Vol. 4, pp. 191203.

Scapens, R. (2006): "Understanding management accounting practices: A personal journey”, The British Accounting Review, Vol. 38, (2006) 1-30.

Scapens, R. y Roberts, J. (1993): "Accounting and control: a case study of resistance to accounting change”, Management Accounting Research, Vol. 4, No. 1, pp. 1-32

Simons, R. (1990): "The role of management control systems in creating competitive advantage: new perspectives", Accounting, Organizations and Society, Vol. 15, No. 1/2, pp. 127-143.

Somohano, F. y Martínez, F. (2003): "Los sistemas de gestión basados en el tiempo: Diseño de un modelo para la producción heterogénea", Revista Española de Financiación y Contabilidad, Vol. XXXII, No. 118, pp. 755-796.

Sriram, R. (1995): “Accounting information system issues of FMS", Integrated Manufacturing Systems, Vol. 6, No. 1, pp. 35-40.

Sulaiman, S. y Mitchell, F. (2005): "Utilising a typology of management accounting change: An empirical analysis", Management Accounting Research, pp. 16 (2005) 422-437.

Street, C. y Meister, B. (2004): "Small business growth and internal transparency: the role of information systems", MIS Quarterly, Vol. 28, No. 3, September 2004, pp. 473-506.

Vélez, M.; Sánchez, M. y Araújo, P. (2005): "La influencia del contable de gestión en los programas de cambio organizativo y contable: evidencia empírica en un entorno organizativo de mejora continua", Revista Española de Financiación y Contabilidad, Vol. XXXIV. Núm. 124, enero-marzo 2005, pp. 77-112.

Williams, J. y Seaman, A. (2002): "Management accounting systems change and departmental performance: the influence of managerial information and task uncertainty", Management Accounting Research, Vol. 13, pp. 419-445. 